\title{
Editorial
}

\section{Mindfulness and the Aging Brain}

With improving maternal and reproductive health in South Asia, physical and mental health of the older women is increasingly becoming the focus. For women, midlife encompasses the stage of menopausal transition starting a few years before the natural menopause that occurs around 51 years of age. Midlife then continues till 65 years of age, the threshold for older adulthood. Women in their midlife are often the primary caregivers for their spouse's parents or less commonly of their own parents who have variable levels of cognitive decline and physical restriction. This brings to fore their desire to avoid similar decline in their older age.

The theme for celebrating the world menopause day in October 2015 is "What comes

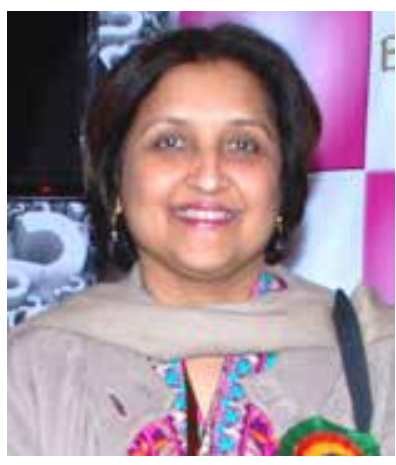
to mind-menopause and the aging brain". During the midlife, modest inattention is noticeable and subtle decline in cognition sets in. However, no evidence of rapid deterioration has been reported in the perimenopause. Pathological processes underlying various forms of dementia are quite distinct from cognitive aging. Some of the risk factors associated with cognitive decline and dementia are nonmodifiable while some are amenable to intervention in the midlife individual. The nonmodifiable factors include early childhood environment, educational attainment and occupation. Similarly marital status is usually not within the individual's control and can change at different ages.

Cognitive decline is often associated with medical disorders like hypertension, diabetes mellitis and hyperlipidemia that contribute significantly to cerebrovascular disease (CVD). These conditions are again more common in the South Asian population. Good control of these medical disorders can assist in secondary prevention of cognitive decline and dementia in the affected individual. Cigarette smoking if prevented could influence the future health of our generations to come. Public health initiatives to assist the smokers to cease smoking are needed from the legislators and advocacy groups.

The modifiable factors that can be addressed at midlife include dietary modification, drugs, vitamins and various mental, physical and social activities. A recently published meta-analysis very well describes the literature review of individually modifiable risk factors for the aging brain. ${ }^{1}$ Although widely used as over the counter preparations, vitamin B and D supplementation, omega 3 polyunsaturated fatty acids, Gingko biloba and DHEA have not shown any benefit for cognition decline. The nurses health study has reported some improvement in cognition in women on long-term Mediterranean diet. ${ }^{2}$ Similarly, another study from Spain has reported improved memory and global cognition on Mediterranean diet with olive oil. ${ }^{3}$

Menopausal hormone therapy (MHT) initiated beyond 60 years has no improvement in mental functions and indeed harms the women. ${ }^{4}$ In the younger postmenopausal women, short-term benefit in cognition is evident if MHT is started at the time of surgical menopause. Results of further ongoing and new trials will clarify its role in the younger postmenopausal women before 60 years of age. Soy Isoflavones improve memory but have no effects on global cognition. ${ }^{1}$

Although aerobic physical activity like walking reduces Alzheimer's risk, improves cardiorespiratory health, it has not been found to improve cognition. Women are twice as likely as men to develop depression. The menopausal transition may be a time of increased vulnerability to depression, but the risk is not elevated in the older late postmenopausal women. Depression should be recognized early and treated. Depression is strongly associated with Alzheimer's disease risk yet its association may not be causal.

To improve brain health and cognition, mentally stimulating leisure activities and social engagement are generally recommended. The advanced cognitive training for independent and vital elderly (ACTIVE) trial supports the logic of 'Use it or lose it' by revealing improved cognition with training for up to 10 years in older adults without pre-existing cognitive impairment. ${ }^{5}$ Tai Chi exercises improved global cognition in contrast to western exercise ${ }^{6}$ and Yoga where no such benefit was found. 
In summary, a holistic approach with healthy life style, cessation of cigarette smoking, increased intake of fish and legumes, social engagement and Tai Chi regimen can give modest benefits in prevention of cognitive decline. Further robust trials into various existing as well as new interventions may guide us for effective strategies to improve mindfulness in older women.

\section{REFERENCES}

1. Lehert P, Villaseca P, Hogervorst E, Maki PM, Henderson VW. Individually modifiable risk factors to ameliorate cognitive aging: a systematic review and meta-analysis. Climacteric 2015 Oct;18(5):678-689.

2. Samieri C, Goldstien F, Rosner BA, et al. Mediterranean diet and cognitive function in older age. Epidemiology 2013 Jul;24(4): 490-499.

3. Valls-Pedret C, Sala-Vila A, Serra-Mir M, et al. Mediterranean diet and age related cognitive decline: a randomized clinical trial. JAMA Intern Med 2015 Jul;175(7):1094-1103.

4. Manson JE, Chlebowski RT, Stefanick ML, et al. Menopausal hormone therapy and health outcomes during the intervention and extended poststopping phases of the Women's Health Initiative randomized trials. JAMA 2013 Oct 2;310(13):1353-1368.

5. Ball K, Berch DB, Helmers KF, et al. Effects of cognitive training interventions with older adults: a randomized clinical trial. JAMA 2002 Nov 13;288(18):2271-2281.

6. Taylor-Piliae RE, Newell KA, Cherin R, Lee MJ, King AC, Haskell WL. Tai Chi and western exercise on physical and cognitive functioning in healthy community dwelling older adults: a randomized clinical trial. J Aging Phys Act 2010 Jul;18(3):261-279.

Syeda Batool Mazhar MBBS (Pb) FRCOG (UK) FCPS (PK)

Head, MCH Centre, PIMS, SZABMU

Islamabad, Pakistan 